additional data on the role played by the elements of low atomic number in the structure of cometary meteroids.

Most meteor spectra secured are of cometary meteroids. Since little is known of the quantitative chemical composition of cometary material, an important problem for the future is how to use meteor spectra to derive chemical compositions of meteroids. The temperature concept, based on considerations of thermodynamical equilibrium, is unreliable for quantitative estimates. Thanks to the recent laboratory determinations of atomic-collision cross sections under conditions that simulate meteor radiation (04.104.012; 06.104.002, 3; Boitnott and Savage, 1972), a start has been made in calculating meteroid compositions (Millman, 1972). But we badly need more laboratory values for the relevant atomic constants, and more good photometry of detailed meteor spectra. Eventually this should lead us to a study of the differences in composition among the meteroids originating from various comets.

Some theoretical work has been attempted in an effort to explain the presence or absence of certain enduring features in meteor spectra, such as that near $3840 \AA$ and the line of neutral oxygen at $5577 \AA$. So far, this effort has been very successful and much remains to be done on the theory, not only of the enduring features, but also on the relation between the ionized lines and the neutral lines and on the relative strengths of the band systems of the light elements in meteor spectra. Note should be taken of the current activity concerned with the theoretical study of molecules in the coma of a comet. Since the meteor vapor originates from solids originally imbedded in the type of solid that produces the comet vapor, progress on research in the two fields should be closely coordinated.

Another important field of investigation that should be considered in this problem of the chemical composition of cometary matter is the mass-spectrometer measures made in the ionosphere with the aid of upper-air rockets (Narcisi, 1971; Goldberg and Aikin, 1971). Interpretation of these measures is bedeviled by the large number of chemical and photochemical processes that establish the measured concentrations of ions. A large amount of data in this field have been accumulated recently, both in North America and in Europe, and it seems highly probable that most of the metallic ions detected are a fallout from meteroids. The frequency of the elements recorded should be correlated with results from meteor spectroscopy, and further correlated with the compositions found for particles of presumed interplanetary dust collected by devices mounted on satellites, rockets, and balloons.

In the various types of observational programs referred to above, the preliminary work on meteoroid composition suggests some affinity between the meteoroids and the carbonaceous chondrites. The big question still remains - what is the quantitative contribution of the lightweight elements and molecules to this picture?

\title{
REFERENCES
}

Boitnott, C. A., Savage, H. F. $\quad$ 1972, Astrophys. Journ., 172, 201.

Goldberg, R. A., Aiken, A. C. $\quad$ 1971, Journ. Geophys. Res., 76, 8352.

Millman, P. M. 1972, Journ. Roy. Astron. Soc. Canada, 66, 201.

Narcisi, R. S. 1971, in F. Verniani, (ed.), Upper Atmosphere Physics, Casa Editrice Compositori, Bologna, 12.

\section{PHYSICAL THEORY OF METEORS}

\section{A. F. Cook}

Development of a viable physical theory of meteors requires a suitable underpinning of observations to keep within reasonable bounds the proliferation of possible detailed processes considered. Optical observations are subject to much more straightforward interpretation than are radar observations, and the smaller the meteoroid the more simple we may hope to find the physical conditions. These considerations lead to the urgent importance of observations of the faintest possible meteors, both with and without dispersion. The best techniques, at present, seem to be those of image intensification and low-light-level television. Other supporting observations are required, 
either optical observations from more than one station or radar observations. Single-station optical observations by television combined with multiple-station radar observations have been made in the United States (Cook et al., Coll. 13). In this case the optical and radar stations were located so that meteors with radiants in or near the television field of view stood a very good chance of being observed at minimum loop ranges by the radar. Single-station observations by television combined with single-station radar observations from the same site have been made in Canada $(05.104 .042,3)$. These have been made with and without gratings in front of the television cameras. Other pertinent spectral observations are reported in the preceding Section.

The importance of the combination of multiple-station radar observations with optical observations lies not only with the determination of the ratio of ionizing efficiency to luminous efficiency but also with the reliable interpretation of radar observations of meteors too faint to be seen optically. So far, no meteor has been seen by both multiple-station radar and television that appears to have been a completely melted single body, held to shape by surface tension and ablating by vaporization. Such a regime appears only to be reached for still fainter meteors accessible only to radar. Accordingly, it is important that radar returns are seen to be limited by rapid ambipolar diffusion of ions and electrons higher than $100 \mathrm{~km}$ above mean sea level and by rapid dissociative recombination of ions and electrons below $80 \mathrm{~km}$ (Southworth, Coll. 13), and that the bulk of the ionization thus appears to be molecular rather than atomic. Also, light curves and apparent ionization curves of individual meteors are accumulating. After the differences between these curves are fully interpreted, a study of the ionization curves of faint radar meteors should bring us face to face with the simplest possible case - that of a meteoroid melted through into a single droplet and vaporizing in free molecular flow.

The meteors accessible to combined television and radar observation appear to provide, at best, some examples of the second simplest cases: (1) meteoroids that have melted through and separated into a cloud of droplets, and (2) a two-stage ablation in which droplets leave by melting and spraying and are subsequently vaporized, not immediately but on a time scale not much shorter than for the ablation of the meteoroid. The faintest spectra observed by image intensification and by lowlight-level television probably come from meteors five orders of magnitude more massive. These correspond to the fainter meteors among those observed by Super-Schmidt cameras. These meteors appear to offer examples of the two-stage ablation process extending down to durations of droplets undergoing vaporization that are much shorter than the duration of the meteor. Both the droplets and the meteoroids may be expected to be in free molecular flow. Experiments in laboratories to determine luminous efficiencies in free molecular flow for individual multiplets have been carried out in the United States (04.104.012;06.104.002, 3; Boitnott and Savage, 1972). These should be continued and expanded to include bands and also ionizing efficiencies. The results may be applied to determination of abundances in meteors and then to prediction of the luminous efficiency in undispersed light. If the ionizing efficiency can also be predicted in this way, a solid base for theory will then exist extending from the faintest radar meteors up through Super-Schmidt meteors.

Only from a fairly complete understanding of the fainter meteors can we proceed confidently to the study of observations of bright photographic meteor up through the brightest fireballs.

Certain guiding principles are emerging for the conduct of investigations: (1) The role of fragmentation should be minimized to avoid its invocation as an excessively simple explanation of what we see. To this end the theory of single bodies melted through into spherical droplets, vaporizing and emitting gray radiation from their surfaces, has been developed in the Soviet Union (Lebedinets, Coll. 13). (2) In the United States this type of approach has been reduced to the fitting of light curves with a family of curves defined by a single parameter. The results show that meteors bright enough to be observed both by television and radar either fragment into a cloud of droplets early in their trajectories or below the two-stage model of ablation. It should be possible to extend this second principle of the use of a family of light curves characterized by a single parameter to the model of the two stages of ablation. (3) The model of a cloud of droplets of sizes limited by aerodynamic load should also be explored. It is apparent that the model will cover the case of initial blending in which shutter breaks on photographs are all filled in and radar returns are hopelessly confused. 
It will also cover the more interesting case of terminal blending at the end of a trajectory where useful information can be found from the earlier part of the trajectory.

The outstanding difficulties to be confronted in pursuit of the general line of attack above are as follows: (1) Single vaporizing-droplet meteoroids appear to occur in the size range accessible only to radar observation. (2) Meteors following the two-stage ablation model (melting and spraying, followed by vaporization of droplets) occur from one extreme, in which the meteoroid is exhausted at a much smaller atmospheric pressure than are the droplets, through one in which the meteoroid is exhausted at about half the pressure at which the last droplets are spent, to one in which the droplets are largely vaporized over a rise in pressure that is small compared with the pressure at the end point. (a) Only one meteor fits the first extreme at present and it is one observed by radar and television. (b) Many of the Super-Schmidt meteors may fall in the intermediate category. (c) A few Super-Schmidt meteors are in the last category.The best possible classification and study of the observations is important here. (3) Larger meteoroids have additional problems caused by viscous flow of the meteoroid in air and pooling of wakes of droplets. (4) For great fireballs this regime passes over to the very complicated one of a detached bow shock ahead of the meteoroid and a boundary layer feeding into the wake. (5) The role of thermal shock should be evaluated. The relatively slow time scale for the aerodynamic flux of heat followed by relaxation of any thermal stresses by melting makes this mechanism unlikely to play an important role in meteors except in the case where a local structural failure suddenly exposes a still-cold surface to the air stream. In such a case, indeed, progressive fracturing, exposing still more material, could lead to a rapidly propagating collapse of the entire meteoroid into dust. (6) Finally, there is the problem of the apparently low crushing strength of many meteoroids. It is possible that such a meteoroid undergoes varying bending while yawing or rotating such that resolution along principal axes of the stress tensor indicates tension in one direction; this results in a significant fraction of the aerodynamic load appearing as a rapidly varying tensile load (R. E. McCrosky, private communication).

\section{REFERENCE}

Boitnott, C. A., Savage, H. F. $\quad$ 1972, Astrophys. Journ., 172, 201.

\section{METEOR ORBITS}

\section{Kresák}

As the final stage of reductions of small-scale photographs recording irregular interactions of meteoroids with the atmosphere, the determination of meteor orbits remains a difficult task with regard to both the extent of the data and their precision. Among recent contributions to the lists of photographic meteor orbits, the greatest share comes from the Harvard Super-Schmidt Program, 1956-59. The number of fireball orbits from the Prairie Network has increased to 235, including the Lost City meteorite of 4 January 1970, the second fall (after Prríbram) for which a photographic orbit is available (05.105.081). The same reference lists eight meteors that, according to their photometric and flight parameters, may be high-density objects; this sample is characterized by low geocentric velocities, low inclinations, and aphelia far inside the orbit of Jupiter. Results from the European All-Sky Network also include several cases of meteorite-like objects, but no find has been reported. The occurrence of three fireballs with low extinction level (Bramberg, $36 \mathrm{~km}$; Buedingen, $31 \mathrm{~km}$; Marburg, $32 \mathrm{~km}$ ) over the German stations during two consecutive nights, 21 and 22 March 1972, is interesting. The orbits computed by Ceplecha and Ježková do not suggest any association among these objects.

On the other end of the mass spectrum, long-term cosmic-dust experiments, including the determination of velocity vectors by multiple-coincidence acoustic sensors, were made on Pioneer 8 and Pioneer 9 spacecrafts. Orbital elements of 16 particles of micron size computed by Berg and Richardson reveal an astonishing abundance of very short periphelion distances $(<0.1 \mathrm{AU})$ and revolution periods of less than $1 \mathrm{yr}$; two orbits are nearly identical with that of the Earth, three are hyperbolic, and only one is similar to those of short-period comets. Two meteoroid velocity meas- 